\title{
Evaluation of Culture Methods for Isolation of Mycobacterium tuberculosis Complex and Its Resistance among Pulmonary Tuberculosis Cases in a Tertiary Care Setting, Kerala - A Pilot Study
}

\author{
Sreeja Nair ${ }^{1}$, Seema Oommen ${ }^{1 *}$ and Vidya Pai $^{2}$ \\ ${ }^{1}$ Department of Microbiology, Pushpagiri Institute of Medical Sciences and Research Centre, \\ Tiruvalla, Kerala, India \\ ${ }^{2}$ Department of Microbiology, Yenepoya Medical College, Yenepoya University, Mangalore, \\ Karnataka, India \\ *Corresponding author
}

\section{Keywords}

Pulmonary tuberculosis (PTB), Mycobacterium tuberculosis complex (MTB complex), Mycobacterium growth indicator tube (MGIT), Lowenstein Jensen (LJ), Drug susceptibility testing (DST)

\section{Article Info}

Accepted: 07 October 2018 Available Online: 10 November 2018

\section{A B S T R A C T}

Tuberculosis is an important public health problem in India and globally. Prompt detection and susceptibility testing of Mycobacterium tuberculosis complex from clinical specimens is essential for appropriate management of patients with tuberculosis. With this in background, this study aimed at the comparison of isolation of Mycobacterium tuberculosis complex from clinical specimens of patients suspected of pulmonary tuberculosis using BACTEC Micro MGIT and Lowenstein Jensen (LJ) media with detection of resistance. A prospective study was carried out for one year from November 2016 to October 2017 in the Department of Microbiology, Pushpagiri Medical College, Tiruvalla, Kerala. A total of 81 samples were processed for Ziehl-Neelsen (Z-N) staining and was then cultured using liquid Mycobacterium growth indicator tube $\left(\mathrm{BD}^{\mathrm{TM}}\right.$ BACTEC $^{\mathrm{TM}}$ Micro MGIT) and solid (Lowenstein Jensen's) media. Isolates confirmed as Mycobacterium tuberculosis complex were subjected to drug susceptibility testing (DST) using modified proportion method by MGIT for 1st line drugs i.e. streptomycin $(1.0 \mu \mathrm{g} / \mathrm{ml})$, isoniazid $(0.1 \mu \mathrm{g} / \mathrm{ml})$, rifampicin $(1 \mu \mathrm{g} / \mathrm{ml})$, ethambutol $(5 \mu \mathrm{g} / \mathrm{ml})$ and pyrazinamide $(100 \mu \mathrm{g} / \mathrm{ml})$. Of the total 81 samples that were cultured, seven $(8.6 \%)$, were positive by both MGIT and Lowenstein Jensen's. Three $(3.7 \%)$ were positive by MGIT only and not by LJ. Only 4/10 (4.9\%) culture positive samples were positive by direct smear examination. Turnaround times for culture positivity by MGIT vs LJ was an average of 15.1 days vs 23.8 days. Sensitivity to all drugs was observed in 4/10 (40\%) isolates. None of these 10 isolates were multidrug resistant. Culturing of the organism in addition to smear, increases the possibility of microbiological diagnosis and offers a possibility for drug susceptibility testing. 


\section{Introduction}

Tuberculosis (TB) caused by Mycobacterium tuberculosis, is a leading health problem worldwide and remains one of the major causes of death from infectious disease. Presently one third of the world population is currently infected with the TB bacillus. According to the Global TB report 2017, there were 2.8 million new cases accounting for one quarter of the world's TB cases (RNTCP., 2018). The emergence of multidrug-resistant TB is increasingly common and is a major health concern in many regions of the world, particularly in developing countries (Balabanova et al., 2009). The long generation time of Mycobacterium tuberculosis complex, (M.tb) delays the rapid diagnosis and drug susceptibility testing. Rapid, accurate diagnosis and drug susceptibility are a key factors to optimize treatment and prevent transmission (Pina-Vaz et al., 2005). Bacteriological confirmed active case detection plays a key role in the diagnosis of tuberculosis (TB). Among the most utilized diagnostics methods; TB smear microscopy still remains the most popular among all available methods in developing countries.

Other commonly used diagnostic technique for the diagnosis of active forms of TB in clinical settings the recently introduced cartridge based TB molecular diagnostic technique has efficient turnaround time and specificity (Cheng VC et al., 2005). Even though various molecular diagnostic tests are available, it does not eliminate the need of conventional microscopy, culture and antitubercular drug sensitivity that are required to monitor the progression of treatment and to detect resistance to drugs other than rifampicin (Anon, 2014). Culture is still required for identification of non-tuberculous Mycobacterium spp. Solid conventional Lowenstein Jensen (LJ) culture method requires at least 6-10 weeks of incubation due to the slow growth rate of the Mycobacterium tuberculosis complex (Gupta et al., 2005). Many studies have demonstrated that the time for detection of mycobacteria with the use of liquid media rather than solid egg or agarbased media is significantly reduced(Abe $\mathrm{C}$, Hosojima, 1992). The automated non radiometric liquid culture methods like the BACTEC mycobacterial growth Indicator tube (MGIT) 960TB system is a state of the art, invitro diagnostic instrument designed and optimized for the rapid detection of mycobacteria from clinical specimens (except blood). This system has a 960-tube capacity for nearly 8000 specimens per year and is useful in laboratories dealing with large specimen loads (Cruciani et al., 2004; Rodrigues et al., 2009). This method has greater turnaround time with good recovery rate than the solid media (Lu et al., 2002).

Semi-automated MGIT (Manual MGIT system - BBL MGIT, Becton Dickinson) system which is based on the same principle and which does not need expensive instrument can be used for the same purpose. The manual system is a lot more affordable for laboratories with a smaller budget (Fadzilah et al., 2009). With this in background, this study aimed at the comparison of isolation of Mycobacterium tuberculosis complex from clinical specimens of patients suspected of pulmonary tuberculosis (PTB) using BD BACTEC $^{\mathrm{TM}}$ Micro MGIT ${ }^{\mathrm{TM}}$ and Lowenstein Jensen (LJ) media and to understand the drug susceptibility patterns of Mycobacterium tuberculosis complex isolated from PTB cases.

\section{Materials and Methods}

A prospective study was carried out for one year from November 2016 to October 2017 in the Department of Microbiology, Pushpagiri Institute of Medical Sciences and Research centre, Tiruvalla and Kerala. The study was 
approved by the institutional ethics committee, dated October 29 2015 (PIMSRC/E1/388A/44/2015). A total of 81 clinical specimens of which 54 were BAL specimens, 20 sputum and seven gastric lavage specimens. The specimens were obtained from suspected cases of pulmonary tuberculosis and from patients who are previously treated for pulmonary tuberculosis from different wards and outpatient departments. These included 64 samples from adults and seven from paediatric patients suspected to have pulmonary tuberculosis. Smears were prepared and stained using ZiehlNeelsen (ZN) staining method and were observed under oil immersion objective of light microscope. All specimens were subjected to culture after decontamination and digestion using $\mathrm{NaLC}-\mathrm{NaOH}$.

The processed samples were inoculated onto the Lowenstein Jensen medium and MGIT tube for liquid culture. A BBL MGIT tube (Becton Dickinson) containing $7 \mathrm{~mL}$ modified middle brook $7 \mathrm{H} 9$ broth was used as a culture medium, to which an enrichment supplement as well as a mixture of antibiotics consisting of polymyxin B, amphotericin B, nalidixic acid, trimethoprim, and azlocillin were added as per manufacturer's instructions. After inoculation, the tubes were incubated at $37^{\circ} \mathrm{C}$.

Tubes were read daily for growth upto 42 days. All the positive tubes were further confirmed by ZN staining and sub-cultured on blood agar plate (to rule out contamination) and a LJ slant (for further identification). Simultaneously along with micro MGIT culture, LJ slants were inoculated with the $0.1 \mathrm{ml}$ of the processed sample and were incubated at $37^{\circ} \mathrm{C}$ for a maximum of eight weeks. They were checked twice weekly for first two weeks and then once every week for maximum period of eight weeks. Bacterial contamination was detected by performing Gram staining from the suspected colony.
The smears prepared from colonies on LJ and MGIT tubes were observed for the presence of acid-fast bacilli with presence of cording which is a characteristic feature of $M$. tuberculosis complex. M. tuberculosis complex isolates were further confirmed using $\mathrm{BD}$ MGIT MTBc identification test (TBc ID). This is a rapid chromatographic immunoassay for the qualitative detection of $M$. tuberculosis complex antigen from AFB smear-positive BD MGIT tubes and culture isolates. The assay was performed according to the manufacturer's instructions.

The confirmed $M$. tuberculosis complex isolates were further subjected to drug susceptibility testing (DST) using the drugs Streptomycin (S) (Isoniazid (I) $(0.1 \mu \mathrm{g} / \mathrm{ml})$, Rifampicin (R) $(1 \mu \mathrm{g} / \mathrm{ml})$, Ethambutol (E) $(5 \mu \mathrm{g} / \mathrm{ml})$ and Pyrazinamide (Z) $(100 \mu \mathrm{g} / \mathrm{ml})$ using the MGIT system as per the manufacturer's protocols. For each sample, five drugs containing MGIT and one control tube without drug were inoculated. The tubes were incubated at $37^{\circ} \mathrm{C}$ and were examined daily for fluorescence with a $365-\mathrm{nm} \mathrm{W}$ light. The results were interpreted only after the growth control tube fluoresced.

\section{Results and Discussion}

Of the total 81 samples cultured, $46(56.8 \%)$ were from males and 35 (43.2\%) from females. Most of our study population were > 50 years (Table 1). Seven (8.6\%) specimens were positive by both MGIT and Lowenstein Jensen's, three (3.7\%) were positive by MGIT only and not by LJ. Only four out of the ten (40\%) culture positive samples were positive by direct smear examination. Turnaround times for culture positivity by MGIT vs LJ was an average of 15.1 days vs 23.8 days. Sensitivity to all drugs was observed in four out of the ten (40\%) isolates (Table 2). Mono resistance was documented to pyrazinamide and streptomycin in 4/10 (40\%) and 1/10 
(10\%) respectively (Table 2$)$. One (10\%) case showed resistance to pyrazinamide and streptomycin both together.

Culturing the organism still remains the cornerstone on which definitive diagnosis of TB relies. It can be performed on a variety of respiratory and non-respiratory samples, is much more sensitive than microscopy and allows the recovery of the bacteria for other studies, such as the identification of mycobacterial species, drug susceptibility testing and genotyping. Liquid cultures generally require a shorter incubation time than culture on solid media for the detection of M. tuberculosis complex. (Morgan et al., 1983) World Health Organization now recommends expanded use of liquid culture systems in resource constrained settings for the diagnosis of TB (World Health Organization, 2007).

The present study demonstrates that recovery of Mycobacterium tuberculosis complex is indeed faster and better in the BACTEC MGIT method compared to conventional methods. The present study demonstrated that micro MGIT system provided better isolation rate of Mycobacterium tuberculosis complex i.e., 10/10 (100\%) from suspected clinical samples than the LJ media which grew 7/10 isolates $(63.95 \%)$. Amongst the ten MGIT positives, $25 \%(5 / 20)$ were from sputum specimens and $9.3 \%$ (5/54) from BAL specimens. Various authors have reported similar findings ranging from 80 to $100 \%$ for MGIT and from 59.7 to $87.2 \%$ for LJ ( $\mathrm{Lu}$ et al., 2002; Alcaide et al., 2000; Hanna et al., 1999). Though liquid culture yields better isolation, for maximum isolation of organisms, the US Centers for Disease Control and Prevention recommends that liquid media should be used in combination with solid media (Kubica and Kent, 1985). Including a solid media along with LJ culture helps in detecting mixed cultures and for correlation with any colony morphologies present (Caulfield and Wengenack, 2016).

Microscopic investigation of sputum is still the most commonly used method for tuberculosis diagnosis. The sensitivity of the microscopic investigation of sputum using the Ziehl-Neelsen staining is $20-60 \%$ and $\geq 80 \%$ in some cases (Çalışkan and Kaya, 1980). In this study, only $4 / 10 \quad(40 \%)$ culture positive samples were positive by direct smear examination. Out of the four smear positive samples, MGIT was positive in all four while LJ medium was positive in only three samples. Even though microscopic investigations are rapid, its sensitivity is relatively low. The success of the microscopic investigation depends on the prevalence of the disease, presence of cavity, and the quality of the sample. While $10^{6} \mathrm{AFB} / \mathrm{ml}$ of sputum usually results in a positive smear, the detection rate drops to $60 \%$ if $10^{4} \mathrm{AFB} / \mathrm{ml}$ are present and to only $10 \%$ if less than $10^{3} \mathrm{AFB} / \mathrm{ml}$ are in the smear (Palomino, 2005). Sputum smears give a negative result for approximately half of the pulmonary tuberculosis patients. Causes of negative results of the sputum smear include insufficiency or mistakes during collection, processing, or microscopic examination of sputum (Caliskan and Kaya, 1980). Furthermore, microscopy will not discriminate between $M$. tuberculosis complex and other mycobacteria, nor viable from dead bacilli. However, in low income settings, where culture facilities are not available, empirical or even antitubercular therapy are initiated based on the positive smear microscopy report (Amit Banik et al., 2018).

Besides higher rates of isolation, the turnaround time for culture positivity by MGIT was an average of 15.1 days vs 23.8 days for LJ in the present study. The average time to detection of mycobacterial growth according to the smear positivity noted to be significantly shorter for MGIT (9.8 days for 
smear positive and 20.4 days for smear negative) compared to LJ media (23.3 days for smear positive and 24.4 days for smear negative). This study also demonstrated that the time to culture positivity depended on bacterial load/smear grading. In samples with microscopy result of 3+, MGIT showed growth in an average of seven days. Similar studies have been reported by S Rishi et al., 2007 which shows that MGIT requires an average of 9.66 days and LJ 28.8 days (Rishi, S., Sinha, P., Malhotra, B. and Pal 2007). Another study done by Somoskovi and Magyar (1999) shows that MGIT required 12.6 days for smear positive sample and 15.8 days for smear negative results. Based on these results detection and identification of most cultures are rapid and reliable when liquid culture system is been used specially in smear negative patients too.

Chances of contamination are more with liquid media. In our study, LJ medium (3/81) showed a lesser contamination rate of $3.7 \%$ compared to MGIT (4/81) with 4.9\% contamination. Similar contamination rates were reported from Iran where the rates were $4.8 \%$ for liquid media (Somoskövi et al.,
2000). Unlike this study, higher contamination rates were reported in a study conducted in Ethiopia were the rates for MGIT and LJ were 9.3\% and $15 \%$ respectively (Diriba et al., 2017). The difference in the rates of contamination could be explained by the difference in the study settings in sample collection, transportation, patient instructions, rejection criteria of the laboratory and difference in sample storage conditions (Siddiqi and Rüsch-Gerdes, 2006).

The common age group affected in this study was the elderly i.e., 51-70 years of age. The results showed that the TB incidence in males was significantly higher than females. Also, the age distribution showed that the TB was the age group of over 65, which was the world's TB epidemic trend (World Health Organization, 2016). Acute or chronic diseases, malnutrition, and the biological changes associated with aging can disrupt protective barriers, impair microbial clearance mechanisms (Rajagopalan, 2001), and contribute to the expected age-related diminution in cellular immune responses. Therefore prompt diagnosis of $\mathrm{TB}$ in the elderly becomes important.

Table.1 Age distribution of cases studied

\begin{tabular}{|l|c|c|}
\hline Age group & $\begin{array}{c}\text { Male (positives) } \\
n=46\end{array}$ & $\begin{array}{c}\text { Female (positives) } \\
n=35\end{array}$ \\
\hline $0-10$ & $4(0)$ & $1(0)$ \\
\hline $11-20$ & $0(0)$ & $0(0)$ \\
\hline $21-30$ & $1(1)$ & $3(0)$ \\
\hline $31-40$ & $2(0)$ & $5(1)$ \\
\hline $41-50$ & $3(1)$ & $3(0)$ \\
\hline $51-60$ & $9(2)$ & $5(0)$ \\
\hline $61-70$ & $18(2)$ & $10(0)$ \\
\hline $71-80$ & $7(1)$ & $6(0)$ \\
\hline $81-90$ & $2(2)$ & $2(0)$ \\
\hline 90 above & $0(0)$ & $0(0)$ \\
\hline
\end{tabular}


Table.2 Drug susceptibility pattern of $M$. tuberculosis complex isolated

\begin{tabular}{|l|}
\hline Pattern of drug resistance \\
\hline Sensitive to all five drugs \\
\hline Resistance pattern \\
\hline To all five drugs \\
\hline Streptomycin only \\
\hline Pyrazinamide only \\
\hline Pyrazinamide \& Ethambutol \\
\hline MDR \\
\hline
\end{tabular}

\begin{tabular}{|c|}
\hline Number $(\%) \mathbf{n = 1 0}$ \\
\hline $4(40 \%)$ \\
\hline 0 \\
\hline $1(10 \%)$ \\
\hline $4(40 \%)$ \\
\hline $1(10 \%)$ \\
0
\end{tabular}

Emergence of multidrug resistant tuberculosis (MDR-TB) makes the control of tuberculosis more difficult. Therefore, the laboratories are challenged to provide rapid antimicrobial susceptibility testing to ensure effective treatment of tuberculosis and thereby preventing further development of drug resistance in the causative strain due to inadequate drug combinations for extended periods of time (Ådjers-Koskela and Katila 2003). Results obtained with the conventional indirect susceptibility testing methods, especially with solid media, become available too late to influence a timely decision on patient management. Most National TB control programmes (NTP) are strengthening their capacity for the detection and treatment of TB, but despite these steps many patients are unconfirmed because relatively few centres are equipped or have the resources to detect TB and conduct DST (Vashishth Mishra et al., 2016). Culturing the organism still remains essential in monitoring therapy and also in performing the DST to detect resistance to drugs other than despite the availability of various molecular methods.

In the present study, drug sensitivity testing (DST) was carried for all first-line antitubercular drugs - streptomycin $(1.0 \mu \mathrm{g} / \mathrm{ml})$, isoniazid $(0.1 \mu \mathrm{g} / \mathrm{ml})$, rifampicin $(1 \mu \mathrm{g} / \mathrm{ml})$, ethambutol $(5 \mu \mathrm{g} / \mathrm{ml})$ and pyrazinamide $(100 \mu \mathrm{g} / \mathrm{ml}$ (Table 2). Out of $10 \mathrm{M}$. tuberculosis complex isolates tested for drug sensitivity against first line drugs, four isolates $(40 \%)$ were sensitive to all the five drugs, while six isolates $(60 \%)$ were found to be resistant to one or more drugs. This is in concordance with the study conducted in Karnataka where $25 \%$ of the isolates were sensitive to all drugs while $75 \%$ were resistant to one or more drugs (Gaude et al., 2015)

The detected streptomycin monoresistance in this study were $10.0 \%$. Similar studies conducted in Ethiopia reported a lower resistance of $7.0 \%$ (Seyoum et al., 2014). Unlike this study, Sethi et al., in 2013, reported that drug resistance to streptomycin was $28.1 \%$. These variations may be because of varied geographical distribution, circulating strain patterns, demographic, and epidemiological difference. None of our isolates were multi-drug resistant (resistance to at least isoniazid and rifampicin). Monoresistance to pyrazinamide were $40 \%$ in this study.

Poly resistance (resistant to two drugs or more except isoniazid and rifampicin combination) to pyrazinamide and ethambutol were observed in $1 / 10(10 \%)$ isolates. The resistance to ethambutol and pyrazinamide the two first line drugs, is not routinely monitored and thus poorly described (Whitfield et al., 2015). Globally the prevalence of pyrazinamide resistance was reported in $16.2 \%$ of the total population of TB patients, 
41.3\% among TB patients at high risk of MDR-TB, and $60.5 \%$ in patients with confirmed MDR-TB (Whitfield et al., 2015).

The major limitation of the present study is the small sample size and therefore, it is not a representation of the population at large. A larger sample size, comparison with molecular detection methods for detecting both positivity and drug resistance followed by epidemiological typing of the isolates would lead to a better understanding of the disease.

In this study it was observed that the overall performance of BACTEC MGIT liquid culture system is found to be better than the conventional LJ methods for rapid recovery of M. tuberculosis complex with shorter turnaround time in both smear positive and negative clinical specimens. The study may not reflect the actual prevalence of the disease burden and resistance patterns in this region, but with a larger sample size and more indepth studies this can be overcome. This would help bring out newer knowledge on epidemiology and help in to devising novel ways to decimate the huge socioeconomic effect of this dreaded disease.

\section{References}

Abe C, Hosojima S, F.Y. et al., 1992. Comparison of MBCheck, BACTEC, and egg-based media for recovery of mycobacteria. J Clin Microbiol, 30, pp.878-881.

Ådjers-Koskela, K. and Katila, M.L., 2003. Susceptibility testing with the manual mycobacteria growth indicator tube (MGIT) and the MGIT 960 system provides rapand reliable verification of multidrug-resistant tuberculosis. Journal of Clinical Microbiology, 41(3), pp.1235-1239.

Alcaide, F. et al., 2000. Evaluation of the
BACTEC MGIT 960 and the MB/BacT systems for recovery of mycobacteria from clinical specimens and for species identification by DNA AccuProbe. Journal of Clinical Microbiology, 38(1), pp.398-401.

Amit Banik, Niladrisekhar Das, Valarie Wihiwot Lyngdoh, A.C.P. and Dut, V., 2018. Prevalence and first-line drug sensitivity trends of Mycobacterium tuberculosis at a tertiary center in North-East India. The Egyptian Journal of Chest Diseases and Tuberculosis, 67:, pp.32-37.

Anon, 2014. International standard for tuberculosis care 3rd editio., Available at: www.who.int/tb/publications/ standards-tb-care-2014/.

Balabanova, Y. et al., 2009. An integrated approach to rapid diagnosis of tuberculosis and multidrug resistance using liquid culture and molecular methods in Russia. PLoS ONE, 4(9).

Çalışkan, T. and Kaya, H., 1980. Smearnegative pulmonary tuberculosis. Tubercle, 61(2), pp.113-115.

Caulfield, A.J. and Wengenack, N.L., 2016. Diagnosis of active tuberculosis disease: From microscopy to molecular techniques. Journal of Clinical Tuberculosis and Other Mycobacterial Diseases, 4, pp.33-43. Available at: http://dx.doi.org/10.1016/j.jctube.2016. 05.005 .

Cheng VC, Yew WW, Y.K., 2005. Molecular diagnostics in tuberculosis. Eur J Clin Microbiol Infect Dis., 24, p.711-20.

Cruciani, M. et al., 2004. Meta-analysis of BACTEC MGIT 960 and BACTEC 460 TB, with or without solid media, for detection of mycobacteria. $J$ Clin Microbiol, 42(5), pp.2321-2325. Available at: http://www.ncbi.nlm. nih.gov/entrez/query.fcgi?cmd=Retrieve $\& \mathrm{db}=$ PubMed\&dopt $=$ Citation\&list_uid $\mathrm{s}=15131224 \% 5 \mathrm{Cnhttp} / / / \mathrm{jcm}$.asm.org/co 
ntent/42/5/2321.full.pdf.

Diriba, G. et al., 2017. Performance of Mycobacterium Growth Indicator Tube BACTEC 960 with Lowenstein-Jensen method for diagnosis of Mycobacterium tuberculosis at Ethiopian National Tuberculosis Reference Laboratory, Addis Ababa, Ethiopia. BMC Research Notes, 10(1), pp.1-6.

Fadzilah, M.N., Ng, K.P. and Ngeow, Y.F., 2009. The manual MGIT system for the detection of $\mathrm{M}$ tuberculosis in respiratory specimens: An experience in the University Malaya Medical Centre. Malaysian Journal of Pathology, 31(2), pp.93-97.

Gaude, G., Praveenkumar and Hattiholli, J., 2015. Drug resistance patterns among pulmonary tuberculosis patients in a tertiary care hospital in northern Karnataka. Journal of Medicine in the Tropics, 17(2), p.81. Available at: http://www.jmedtropics.org/text.asp?20 15/17/2/81/161510.

Gupta, S. et al., 2005. Comparison of the conventional diagnostic modalities, bactec culture and polymerase chain reaction test for diagnosis of tuberculosis. Indian Journal of Medical Microbiology, 23(1), p.29. Available at: http://www.ijmm.org/text.asp?2005/23/ 1/29/13869.

Hanna BA, Ebrahimzadeh A, Elliot LB, M.M. and Novak SM, Rusch-Gerdes S, et al., 1999. Multicentre evaluation of BACTEC MGIT 960 system for recovery of Mycobacteria. $J$ Clin Microbiol, ;37:, p.748-52.

Kubica, G. and Kent, K., 1985. Public health mycobacteriology: a guide for the level III laboratory. Centers for Disease Control, U.S. Department of Health and Human Services, Atlanta, GA, pp.6063.

Lu, D., Heeren, B. and Dunne, W.M., 2002. Comparison of the automated
Mycobacteria Growth Indicator Tube system (BACTEC 960/MGIT) with Löwenstein-Jensen medium for recovery of mycobacteria from clinical specimens. American Journal of Clinical Pathology, 118(4), pp.542545.

Morgan, M.A., Horstmeier C.D., DEYoung D.R., R.G.D., 1983. Mycobacteria from Smear-Negative Specimens. Journal of Clinical Microbiology, 18(2), pp.384388.

Palomino, J.C., 2005. Nonconventional and new methods in the diagnosis of tuberculosis: Feasibility and applicability in the field. European Respiratory Journal, 26(2), pp.339350.

Pina-Vaz, C., Costa-De-Oliveira, S. and Rodrigues, A.G., 2005. Safe susceptibility testing of Mycobacterium tuberculosis by flow cytometry with the fluorescent nucleic acid stain SYTO 16. Journal of Medical Microbiology, 54(1), pp.77-81.

Rajagopalan, S., 2001. Tuberculosis and Aging: A Global Health Problem. Clinical Infectious Diseases, 33(7), pp.1034-1039. Available at: https://academic.oup.com/cid/articlelookup/doi/10.1086/322671.

Revised National Tuberculsois Control programme, 2018. India TB Report. annual status report.

Rishi, S., Sinha, P., Malhotra, B. and Pal, N.I.J. of M.M. 2007; 25(4): 383-386, 2007.. A Comparative Study for the Detection of Mycobacteria by BACTEC MGIT 960, Lowenstein Jensen Media and Direct AFB Smear Examination,. Indian Journal of Medical Microbiology., 25(4), pp.383-386.

Rodrigues, C. et al., 2009. Evaluation of the bactec MGIT 960 TB 0 system for recovery and identification of Mycobacterium tuberculosis complex in 
a high through put tertiary care centre. Indian Journal of Medical Microbiology, 27(3), p.217. Available at: http://www.ijmm.org/text.asp?2009/ 27/3/217/53203.

Seyoum, B. et al., 2014. Prevalence and Drug Resistance Patterns of Mycobacterium tuberculosis among New Smear Positive Pulmonary Tuberculosis Patients in Eastern Ethiopia., 2014.

Siddiqi, S. and Rüsch-Gerdes, S., 2006. Foundation for Innovative New Diagnostics (FIND). MGIT Procedure Manual.

Somoskovi, A. and Magyar, P., 1999. Comparison of Mycobacteria Growth Indicator Tube with MB Redox, Lowenstein-Jensen, and Middlebrook7H11 Media for the Recovery of Mycobacteria in Clinical Specimens. Journal of Clinical Microbiology, 37, pp.1366-1369.

Somoskövi, Á. et al., 2000. Comparison of recoveries of Mycobacterium tuberculosis using the automated BACTEC MGIT 960 system, the BACTEC 460 TB system, and
Lowenstein- Jensen medium. Journal of Clinical Microbiology, 38(6), pp.23952397.

Vashishth Mishra, Hiba Sami, Rajesh Bareja, Rahul Kumar Goyal, R.N.B. and P.D., 2016. Evaluation of MGITover other phenotypic methods for the detection of pulmonary and extrapulmonary TB at a tertiary care centre in North India. IJPSR, 7(6), pp.2568-2572. Available at: e-issn: 0975-8232; P-ISSN: 23205148.

Whitfield, M.G. et al., 2015. A global perspective on pyrazinamide resistance: Systematic review and meta-analysis. PLoS ONE, 10(7), pp.1-16.

World Health Organization, 2007. The use of liquid medium for culture and drug susceptibility testing (DST) in low- and mediumincome settings. Summary of the expert group meeting on the use of liquid culture systems,

World Health Organization, 2016. WHO Report 2016: Global Tuberculosis Control; 2016.

\section{How to cite this article:}

Sreeja Nair, Seema Oommen and Vidya Pai. 2018. Evaluation of Culture Methods for Isolation of Mycobacterium tuberculosis Complex and Its Resistance among Pulmonary Tuberculosis Cases in a Tertiary Care Setting, Kerala - A Pilot Study. Int.J.Curr.Microbiol.App.Sci. 7(11): 452-460. doi: https://doi.org/10.20546/ijcmas.2018.711.053 